\title{
Using "Click-e-Bricks" to Make 3D Elastomeric Structures
}

\section{Citation}

Morin, Stephen A., Yanina Shevchenko, Joshua Lessing, Sen Wai Kwok, Robert F. Shepherd, Adam A. Stokes, and George M. Whitesides. 2014. "Using 'Click-e-Bricks' to Make 3D Elastomeric Structures." Advanced Materials 26 (34) (July 30): 5991-5999. doi:10.1002/ adma.201401642.

\section{Published Version}

doi:10.1002/adma.201401642

\section{Permanent link}

http://nrs.harvard.edu/urn-3:HUL.InstRepos:16883003

\section{Terms of Use}

This article was downloaded from Harvard University's DASH repository, and is made available under the terms and conditions applicable to Open Access Policy Articles, as set forth at http:// nrs.harvard.edu/urn-3:HUL.InstRepos:dash.current.terms-of-use\#OAP

\section{Share Your Story}

The Harvard community has made this article openly available.

Please share how this access benefits you. Submit a story.

\section{Accessibility}




\title{
Using “Click-e-Bricks” to Make 3D Elastomeric Structures
}

\author{
Stephen A. Morin, Yanina Shevchenko, Sen Wai Kwok, Robert F. Shepherd, Adam A. Stokes, \\ Joshua Lessing, and George M. Whitesides*
}

Dr. S.A. Morin, Dr. Y. Shevchenko, Dr. S.W. Kwok, Dr. R.F. Shepherd, Dr. A.A. Stokes, Dr. J. Lessing, Prof. G.M. Whitesides

Department of Chemistry and Chemical Biology

Harvard University

12 Oxford Street, Cambridge, MA 02138, USA

Email: gwhitesides@gmwgroup.harvard.edu

Prof. G.M. Whitesides

Kavli Institute for Bionano Science \& Technology

Harvard University

29 Oxford Street, Cambridge, MA 02138, USA

Prof. G.M. Whitesides

Wyss Institute for Biologically Inspired Engineering

Harvard University

60 Oxford Street, Cambridge, MA 02138, USA

Keywords: soft machine, soft robot, soft actuator, digital manufacturing, and 3D printing. 
Structures composed largely of elastomeric polymers (and composites based on them) are the components that make up soft actuators, ${ }^{[1-3]}$ devices, ${ }^{[4]}$ and machines. ${ }^{[1,5-13]}$ Structures that are planar or quasi-planar (for example the pneumatic networks, or "pneu-nets," that serve as actuators for many soft machines, ${ }^{[7,8]}$ gripers, ${ }^{[1]}$ and robots ${ }^{[7,10,12]}$ ) can be easily fabricated by molding thermosetting elastomers against masters made using a three-dimensional (3D) printer. $^{[1,14,15]}$ Although 3D printing is a versatile methodology, ${ }^{[14-16]}$ it is either not applicable, or impractical, as a method of making many types of 3D structures (especially in elastomeric materials); and even when applicable, it may be difficult or slow as a fabrication method. It is also complicated to use in making composite structures; especially when components of the composite (e.g., sheets of fabric or fiber) cannot be printed. We wished to develop a flexible method for fabricating 3D, composite structures in elastomers that circumvented the limitations of existing methods. We are especially interested in methods that can be used in rapid prototyping.

The approach we describe here uses elastomeric bricks—universal construction elements fabricated from elastomeric polymers - that can be clicked together to assemble many different elastomeric structures. We focused, for illustration, on rectilinear elastomeric bricks that had pegs and recesses similar to those used by Lego ${ }^{\circledR}$ bricks: these features ensured the bricks were properly aligned. This approach is, however, not limited to this design, and many other shapes (e.g., tetrahedrons and other non-orthogonal polyhedra) and types of connections (e.g., ballsocket and other snap connectors) are possible. We fabricated the click-fit elastomeric bricks ("click-e-bricks") using a single master, and assembled them into structures by hand.

Click-e-bricks make it possible to fabricate complex three-dimensional elastomeric structures that would be difficult or impossible to fabricate directly by molding (e.g., those with overhangs 
that contain more than one type of polymer, or those with non-moldable materials) or by $3 \mathrm{D}$ printing. In designing prototypes, these structures can be assembled, examined, tested, and disassembled, and the click-e-bricks can be reused to build new/different structures; if appropriate, liquid pre-polymer can be applied to all or some of the interfaces between click-ebricks, and cured, to make the assembly permanent and to construct airtight channels for pneumatic or hydraulic actuation. The ready availability of click-e-bricks, and of many other component-based building systems (tiles, rod-and-connector assemblies, and others), reduces the difficulties of making architecturally complicated — in terms of material and geometry—soft structures and machines, ${ }^{[1,7,8]}$ and enables users efficiently to design, prototype, and evaluate a wide range of new soft structures and actuators.

Click-e-bricks thus demonstrate a conceptually new approach to the fabrication of elastomeric structures, based on assembly of components fabricated using a single (or limited number of) master components (of one or multiple types). This strategy makes functional soft structures - including ones with complicated internal 3D architectures, composite structures, and non-printable components - easy to make, and because the structures can be assembled and disassembled, it is especially useful in rapid cycles of prototyping. We believe it is particularly appropriate for pneumatically actuated soft machines. ${ }^{[1,2,7-10,12]}$ We illustrate this strategy by assembling click-e-bricks to form simple soft structures and actuators with different sizes and shapes, and with internal channels that enable functions different from pneumatic actuation.

Most machines and structures (broadly defined) consist of a group of interconnected, interacting, or interdependent elements that together generate function. Examples of such systems, constructed of common elements, include buildings (which are constructed using bricks, beams, girders, windows, doors, electrical wiring, plumbing, networks, etc.) and 
automobiles (which use windows, sheet metal parts, gears, axels, wheels, electronic systems, and other standardized components). Here we focus on soft systems built from components with simple geometries and different mechanical properties that together give rise to useful mechanical behavior (e.g., actuation); we focus on elastomeric materials, since they are centrally important in the fabrication of soft systems of all types.

In mechanical systems, numerous varieties of "connectors" have been developed to hold individual elements together. For example, Lego ${ }^{\circledR}$ bricks use a "snap" connector; other widely used connectors include: ball/socket (as found in trailer hitches), hook/loop (as found in Velcro ${ }^{\circledR}$ ), interdigitated teeth (as found in zippers), and rods/connectors (as found in molecular modeling sets). Connectors insure proper function of a system by maintaining the appropriate architecture, and because many connectors are reversible, they enable easy modification of the system. Glues/adhesives/cements are used to reinforce connectors mechanically or, to form a permanent connection. By combining the concept of mechanical connection with brick-based construction, click-e-bricks form the basis for a broadly applicable method of fabrication of soft structures (and soft/hard composites).

Additive manufacturing (3D printing) is another technology that is being applied to the fabrication of 3D soft structures and soft/hard composites. ${ }^{[14-17]}$ The most advanced systems (Stratasys ${ }^{\circledR}$ Polyjet technology, for example ${ }^{[18]}$ ) can print structures using thermoplastics and elastomer "mimics," and combinations of them, but the accessible range of materials properties (mechanical, optical, electrical, etc.) is limited, the build-volume is determined by the size of the instrument, and the process is slow (relative to the assembly of pre-fabricated components). Further, the elastomer "mimics" used are acrylic-based polymers that are significantly harder (shore hardness of A35 at minimum, based on ASTM D2240 standard ${ }^{[18]}$ ) and less extensible 
(maximum $170 \%$ elongation at breakage ${ }^{[18]}$ ) than the silicone polymers (shore hardness of 0030 , based on ASTM D2240 standard, and 900\% elongation at breakage ${ }^{[19]}$ ) we use for fabricating click-e-bricks; This difference in properties limits the usefulness of the harder materials in actuation.

Functional soft structures have also been created by combining voxels_-volumetric pixels $^{[20,21]}$ _ fabricated from soft and hard materials. ${ }^{[22,23]}$ This approach uses evolutionary algorithms to generate — or "evolve," via the mutation of some starting design — a series of voxelbased structures for a specific task (e.g., locomotion). The goodness, or "fitness," of each design is evaluated based on its ability to solve the desired task (e.g., move successfully to a target position), and the best candidates are then fabricated by stacking the necessary voxels, layer-bylayer. This approach requires that the voxels be small enough (that is, have high enough resolution) to form an apparently continuous structure that captures the desired motion/function. Structures may therefore require several thousand voxels, and fully automated assembly (or combinations of automatic and manual assembly) may be required for fabrication.

We have demonstrated a variety of soft machines and robots comprised of pneumatic, bending actuators (pneu-nets) arranged into architectures that enable function. ${ }^{[1,7-10,12]}$ We have fabricated pneu-nets using soft lithography ${ }^{[24]}$ : that is, by casting thermosetting silicone elastomers against masters (usually) created using 3D printing. ${ }^{[1]}$ The roughly planar castings, which have the inverse of the topology of the masters, are stacked and sealed using liquid prepolymer glue to yield functional devices. This approach has successfully generated soft robots capable of gripping ${ }^{[1]}$ manipulation, ${ }^{[9]}$ locomotion, ${ }^{[7,12,25]}$ and concealment by camouflage, ${ }^{[8]}$ and of functions that combine these capabilities. ${ }^{[8,12]}$ There remain designs, especially those that combine multiple materials in $3 \mathrm{D}$ configurations, that are difficult or impossible to create using 
only soft lithography. Here we expand the types of 3D soft and soft/hard composite structures that can be easily made, emphasizing those generally useful to the field of soft machines.

Once the correct design of a "brick" - the elementary building block— has been settled upon, the bricks themselves can easily be made from many different materials by molding. The choice of material depends upon the intended function of the structures to be built from the bricks. Masonry bricks are rigid and are mostly used for structural support. By using elastomeric polymers as the materials for fabricating bricks — instead of rigid materials such as stone - we can integrate new properties, especially flexibility (e.g., the ability to bend, twist, and stretch) into the final assembly.

We fabricated rectilinear elastomeric bricks with peg/recess click connectors that were similar in function to- but different in important respects of design, fabrication, materials, and use from-Lego ${ }^{\circledR}$ bricks. ${ }^{[26]}$ The most significant of these differences is the design of the click-ebrick recesses, which are cavities equal in size and shape to the pegs that fit into them. In contrast, Lego ${ }^{\circledR}$ bricks do not have recesses that inversely replicate the topography of the pegs; instead, the pegs in Lego ${ }^{\circledR}$ bricks snap between the external walls of the bricks and sets of hollow cylinders located inside the bricks. The peg/recess design used for click-e-bricks would not work well with the rigid thermoplastic (acrylonitrile butadiene styrene, ABS) used in Lego ${ }^{\circledR}$ bricks because this material does not deform (e.g., stretch, flex, compress) easily. Also, click-ebricks have solid interiors, while Lego ${ }^{\circledR}$ bricks are mostly hollow.

We chose a click-e-brick design (see Supporting Information (SI), Figure S1) based on the peg/recess connection because: (i) This type of connection is simple, reliable, and reversible. (ii) The elastomeric bricks can be clicked together, using one or more pegs, depending on the structures. (iii) All elastomeric bricks are a two-dimensional array of the same elementary 
peg/recess connector (e.g., $1 \times 1,1 \times 3$, or $2 \times 3$ ). The last point is important for fabrication, because one large click-e-brick (e.g., a large $6 \times 9$ array, or a continuous sheet produced by reel-to-reel casting) can be cut to yield smaller click-e-bricks, or to create vertical through-holes, or generate special purpose, non-standardized shapes. ${ }^{[26]}$ Aligning and stacking click-e-bricks with throughholes is a simple way to generate channels in extended 3D structures. ${ }^{[26]}$

For applications where the structures assembled from click-e-bricks need to transport or hold fluids, the blocks must be glued together to prevent leakage of gas or liquid. We connected click-e-bricks permanently with liquid pre-polymer adhesives that, once cured, had mechanical strength similar to that of the bricks, and that generated structures that were indistinguishable, visually, from a single casting. ${ }^{[26]}$ The ability to connect bricks permanently or reversibly is a very useful feature of this approach.

We actuated the click-e-brick-based structures by inflating them, through a single inlet tube, with low pressure $(<100 \mathrm{kPa})$ air. As a fluid, air is nearly inviscid, readily available, and does not significantly change the mass of the pressurized structure. This type of actuation was intended only to illustrate the sets of motion that can be achieved. We have not tried to optimize the structures, or to use other fluids (e.g., Newtonian and non-Newtonian liquids, suspensions of granular solids, photocurable polymers, low-melting alloys, and others).

The click-e-bricks we describe here can be thought of as two-dimensional arrays of the same elementary unit (see SI, Figure S1A, B). This elementary unit consists of a cylindrical peg that is directly above a cylindrical recess designed to mate with the peg of another click-e-brick. Some or all of the pegs/recesses can be used to connect the click-e-bricks together (see SI, Figure S1C). To fabricate click-e-bricks we used a large master mold (fabricated using 3D printing) that forms a $6 \times 9$ rectangular array of the elementary brick units. We filled the mold with a single material 
for homogeneous click-e-bricks, or layered different materials into the mold to create click-ebricks (for example, to generate pegs/recesses that had mechanical properties different from that of the body; see SI, Figure S2). ${ }^{[26]}$ To generate click-e-bricks of various dimensions and numbers of pins, we cut the master click-e-brick into smaller dimensions using a razor blade (see SI, Figure S3). ${ }^{[26]}$ The ability to create click-e-bricks of desired (and arbitrary, in the plane of the original sheet) dimensions by cutting a master click-e-brick accordingly is another useful feature of soft elastomers.

To prototype soft structures with click-e-bricks, we assembled them into basic structures; when appropriate, we disassembled them and reused the same click-e-bricks to build different structures. Once a structure with the desired characteristics was built, we fabricated the final, permanent version by "gluing" together the click-e-bricks. Following this approach we fabricated a set of structures that demonstrate the four most basic mechanical deformations possible with click-e-bricks (Figure 1): the click-e-bricks can be bent (Figure 1A, B), twisted (Figure 1C), stretched (Figure 1D), and forced to accommodate incompatibilities in the registration of the pegs (e.g., an extra plane; Figure 1E). For prototyping, we used composite click-e-bricks with pegs/recesses that were made of polydimethylsiloxane (PDMS) and bodies that were made of Ecoflex ${ }^{\circledR}$ (where PDMS is the slightly stiffer material, see SI, Figure S2) ${ }^{[26]}$ Click-e-bricks with stiffer pegs and recesses were more easily assembled and disassembled than click-e-bricks with soft and flexible pegs/recesses made entirely of Ecoflex ${ }^{\circledR}$. The final structures (Figure 1) were fabricated from Ecoflex ${ }^{\circledR}$-only click-e-bricks that were glued together using Ecoflex ${ }^{\circledR}$ pre-polymer. $^{[26]}$

It was also possible to combine more than one deformation in a single structure. For example, by bending and twisting strips of click-e-bricks, we created a circular bilayer with a full 
Figure 1. Structures that illustrate the ability of click-e-bricks to be bent (A, B) twisted (C), stretched (D), and forced to accommodate extra planes (E). In all cases the photographs are accompanied by a schematic illustration showing the types and numbers of click-e-bricks used in the structure. (A, B) Two different examples of planar walls that can be bent and joined at their ends to form cylinders. The size and curvature of the cylindrical walls can be adjusted by combining click-e-bricks of different dimensions. In each case the axis of bending is parallel to the peg/recess axis. (C) A string of click-e-bricks is held vertically by a clamp and twisted $720^{\circ}$. The axis of twisting is perpendicular to the peg/recess axis. (D) A string of click-e-bricks is stretched perpendicular to the axis of the peg/recess. (E) An extra click-e-brick is inserted between two layers to create an extra plane. (F) A click-e-brick ring with a full- $360^{\circ}$ twist. (G) Structure consisting of two arches. The axes of bending are perpendicular to the peg/recess axis. All scale bars are $1 \mathrm{~cm}$. 
Figure. 1.
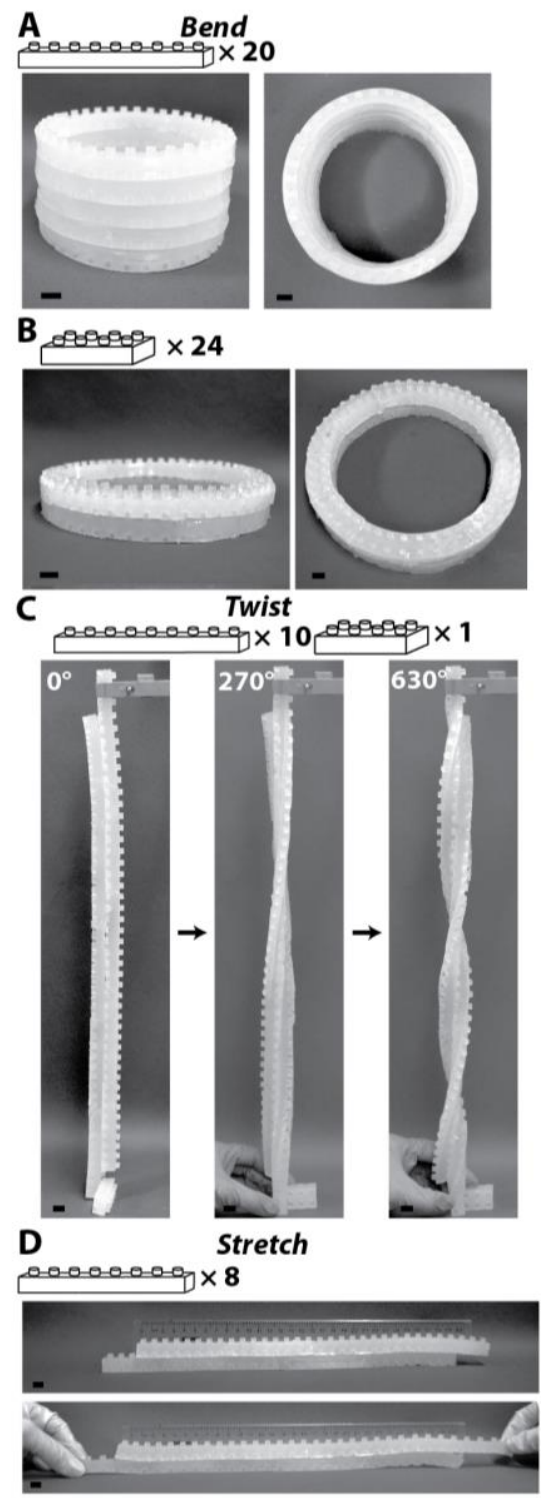

E Extra Plane

0-000-000-9 $\times 8$ $0-0-0-0 \times 1 \times 1$

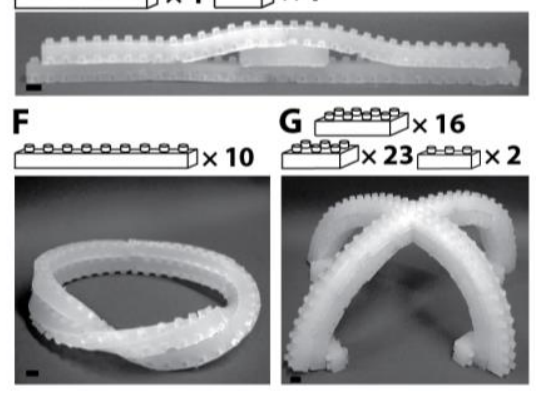


$180^{\circ}$ of twist along its circumference (Figure 1F). Mobius structures are also possible and only $0^{\circ}, 90^{\circ}, 180^{\circ}$, or $270^{\circ}$ of twist are needed. In addition, by bending click-e-bricks about axes orthogonal to the axis of the peg/recess (Figure 1A), we created a self-supporting structure from two arches (Figure 1G).

We glued a specific set of click-e-bricks together with liquid PDMS pre-polymer to create inflatable structures (Figure 2). ${ }^{[26]}$ For this function the glue was necessary to create air-tight seals between the click-e-bricks. We began with hollow cubes, because they are the simplest hollow structures to fabricate using rectangular click-e-bricks. The cubes were assembled layerby-layer (Figure 2A, B) using a design that ensured that the vertical seams of each layer did not line up. This type of registration was important for creating cubes with air-tight vertical edges.

We controlled the direction and magnitude of expansion by patterning click-e-bricks of different Young's moduli into the walls of the cubes. When we pneumatically actuated a hollow cube assembled from four layers of Ecoflex ${ }^{\circledR}$ click-e-bricks, and a bottom click-e-brick made of PDMS, the cube expanded into a spheroid pinned by the PDMS base (Figure 2A-C, see SI, Video S1; here we added fluorescent dye to the PDMS pre-polymer used to cast the click-ebricks). The fluorescence of the click-e-bricks (under illumination with UV light) highlights their deformation during actuation—click-e-bricks do not need to be colored, but this property could be useful when it is necessary to differentiate the bricks optically (Figure 2A-C). We fabricated different cubes by replacing portions of the three middle layers - fabricated entirely from Ecoflex ${ }^{\circledR}$ click-e-bricks in the previous cubes—with click-e-bricks made of PDMS. Although these structures shared the same cubic shape when not actuated, they expanded into different shapes (e.g., a wedge, see SI, Figure S4A-C, Video S2; and an elongated rectangle, see SI, Figure S4D-F, Video S3) when inflated pneumatically, where the anisotropic expansion 
Figure 2. Assembly of click-e-bricks into hollow structures and their pneumatic actuation. (A, D) Schematic illustration of the click-e-bricks used for fabricating the structures shown in panel C and panel G. Gray is PDMS and white is Ecoflex ${ }^{\circledR}$. (B) Schematic illustration of the layer-bylayer assembly of the cube in C. (E-G) Schematic illustration of the click-e-brick layers as viewed from the top (E) and the stacking of these layers $(F)$ for fabricating the cross-shaped structure shown in G. (C, G) Click-e-brick-based actuators when no net pressure was applied to the interior of the hollow structures (top panels), and when a net positive air pressure was applied (bottom panels). In all cases, pressurized air was applied via a single tube routed through one of the walls of each structure, and the click-e-bricks were glued together by applying and curing liquid PDMS or Ecoflex ${ }^{\circledR}$ pre-polymer at their interface. All scale bars are $1 \mathrm{~cm}(\Delta \mathrm{P}=40 \mathrm{KPa} \sim$ 0.4 atm of applied pressure). 
Figure 2.

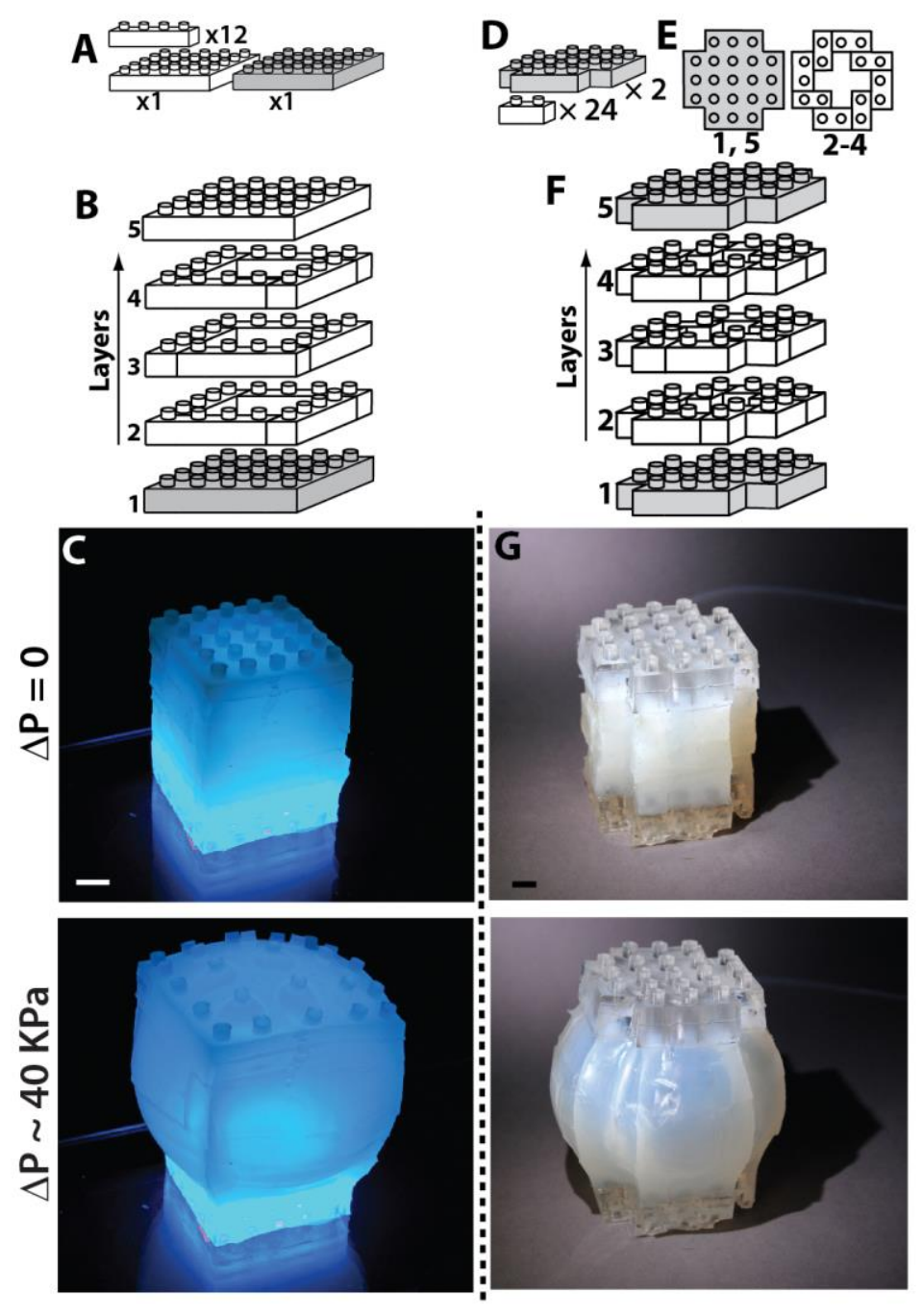


depended on the organization of the click-e-bricks in each layer. This approach can be extended to the fabrication of other rectilinear structures. For example, we constructed a cross-shaped structure (Figure 2D-E) by gluing together a set of rectangular click-e-bricks. When pressurized, the cross-shaped structure expanded into a convex cylinder (Figure 2G, see SI, Video S4).

We also created more complex multi-chamber systems (which approached some of the desired functionalities of soft machines) using click-e-bricks. For instance, we cut out portions of click-e-bricks (Figure 3A, B), aligned, stacked, and glued them together to form independently addressable chambers within the assembled structure (Figure 3C). ${ }^{[26]}$ Using this approach, we constructed a tentacle-like soft machine with four independent chambers (Figure 3C) and demonstrated bending motion with four degrees of freedom in three-dimensional space (Figure 3D, see SI, Video S6).

We can incorporate overhangs into these structures that would be difficult to produce in a single molding step. To illustrate this concept we fabricated structure with an overhang protruding from each of its vertical faces (Figure 4A, see SI, Video S7). These overhangs may be used as attachment points for click-e-bricks with other properties or other geometries (Fig. 4B; Video S8). Overhangs also enable complex architectures that combine inflatable structures of different shapes, with distinct motions, by providing multiple sites for overlapping connection. They may also provide positions for reversible structural additions even if the majority of the click-e-bricks in the structure are permanently connected.

Overhangs can also be used to secure deformed rectangular click-e-bricks, into non-reticular confirmations for fabricating structures of different shapes. For example, we assembled three walls of a cube as before, but instead of completing the structure with a fourth wall (satisfying the natural geometry of the rectangular click-e-bricks), we bent the incomplete edges together 
Figure 3. Assembly of click-e-bricks to form a multi-chamber soft machine and its operation. In all cases the click-e-bricks were glued together by curing the liquid PDMS or Ecoflex ${ }^{\circledR}$ prepolymer adhesives applied at their interfaces. Click-e-bricks made of PDMS or Ecoflex ${ }^{\circledR}$ are colored gray and white respectively. (A-C) Schematics illustrating the assembly of the device. (A) Schematic illustration of the click-e-bricks used for assembling the device. Six of the clicke-bricks had four rectangular cutouts (dotted boxes labeled 1 to 4 in panel B, top view) that formed continuous chambers when stacked as shown in panel C. (D, i-viii) Operation of the soft machine. Inset at the upper left corner of each panel shows the state of pressurization of the four chambers. A black chamber is pressurized $(\Delta \mathrm{P} \sim 40 \mathrm{KPa})$ and a white chamber is not. Tubes routed through the side-walls of the device provided access to the internal chambers for delivering and venting of pressurized air. Each chamber required a single tube for pneumatic actuation. All scale bars are $1 \mathrm{~cm}$. 
Figure 3.

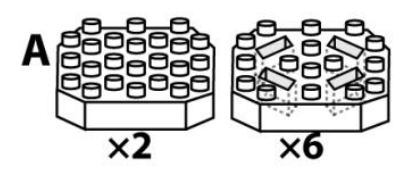

B
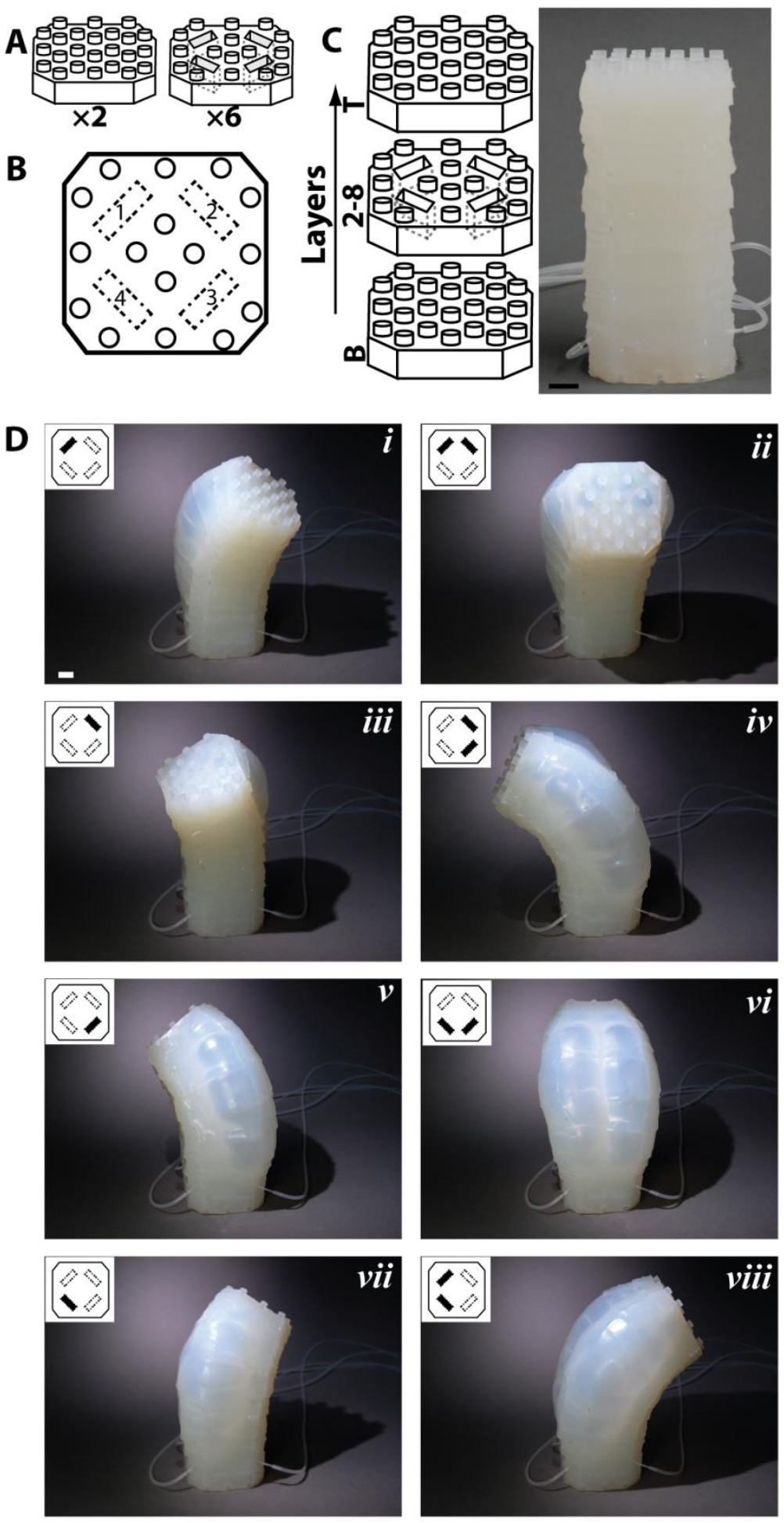
Figure 4. Assembly of click-e-bricks into hollow cubes with overhangs. (A, B) Schematics illustrating the assembly of a cube with overhangs incorporated into its side walls. The click-ebricks that form the overhangs are colored grey. (C) Photo of the assembled device with purple click-e-brick overhangs. (D) Schematic showing the attachment of click-e-bricks to the overhangs and (E) a photo of the corresponding structure. The entire structure is made of Ecoflex ${ }^{\circledR}$ click-e-bricks. Upon inflation (C, E, bottom panels), the structure expands, and the overhangs tilt down. All scale bars are $1 \mathrm{~cm}$. 
Figure 4.

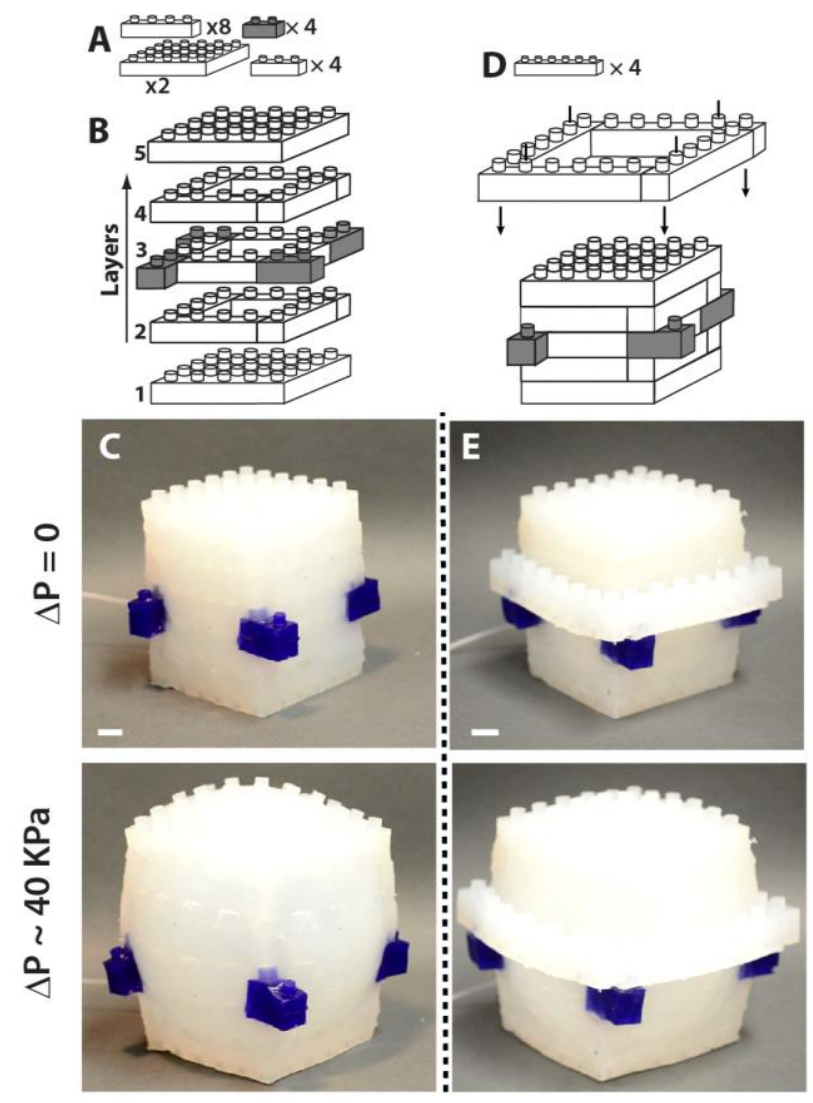


and used the overhangs to secure them to one another (and thus formed a prism, Figure 5A-D). When pressurized the prism expanded into an ellipsoid (Figure 5E, see SI, Video S5).

We are not limited to rectangular click-e-bricks, and it is easy to extend our approach to click-e-bricks of different shapes such as circular click-e-bricks (Figure 5F-H). Using these structures, and incorporating in each layer a brick with greater stiffness than the others, we fabricated a cylindrical structure that both elongated and twisted upon pneumatic pressurization and inflation (Figure 5I). The structure was made of layers of Ecoflex ${ }^{\circledR}$-based click-e-bricks with PDMS-based click-e-bricks inserted in each layer such that they formed a helical path along the vertical wall of the structure (Figure 5H, see SI, Video S9). This demonstration further illustrates how heterogeneous material architecture and (internal in this case) overhangs can generate complex motion.

This paper demonstrates a versatile method — using click-e-bricks — that enables the fabrication of 3D, composite structures in elastomeric polymer that would be difficult, time consuming, or impossible to generate using printing or molding techniques. The approach, which relies on the combination of pre-fabricated click-e-bricks with different properties, is a simple way to achieve these structures, even when complex architectures (e.g., overhangs) are required, because it decouples the details of geometry and composition associated with the final structure from the constraints imposed by the techniques used to generate the click-e-bricksprinting and molding in this case — but the same could be said regardless of the exact method.

Click-e-brick-based fabrication makes possible the exploration of soft structures and actuators useful to the functions of soft devices and machines that were previously inaccessible. These structures can be rapidly assembled, reconfigured, and (if required for their operation) made permanent by "gluing" together the click-e-bricks; this ease of fabrication enables rapid 
Figure 5. Non-rectilinear structures and their pneumatic actuation. (A-D) Schematic illustration showing the click-e-bricks required for and the layer-by-layer assembly of a hollow prism structure. (E) Photos of the click-e-brick-based prism when no pressure or vacuum is applied (top) and when positive air pressure is applied (bottom). The scale bar is $1 \mathrm{~cm}$. (F) Circular click-e-bricks: all Ecoflex ${ }^{\circledR}$ (bottom) and combination Ecoflex ${ }^{\circledR}$ and PDMS (top). (G, H) Schematic illustration of a set of circular click-e-bricks and their assembly into a cylindrical structure. The PDMS sections following a helical path along the vertical wall of the structure. For simplicity, layers 9-15, which are the same as $2-8$, and the purple overhangs of the final structure, are not shown in the schematic. (I) Actuation of the assembled and sealed structure using $100 \mathrm{kPa}$ compressed air. The purple overhangs on the vertical wall help to visualize the twisting motion of the structure. One of the overhangs is annotated with a dotted line. The unactuated structure is $17 \mathrm{~cm}$ tall. 
Figure 5.

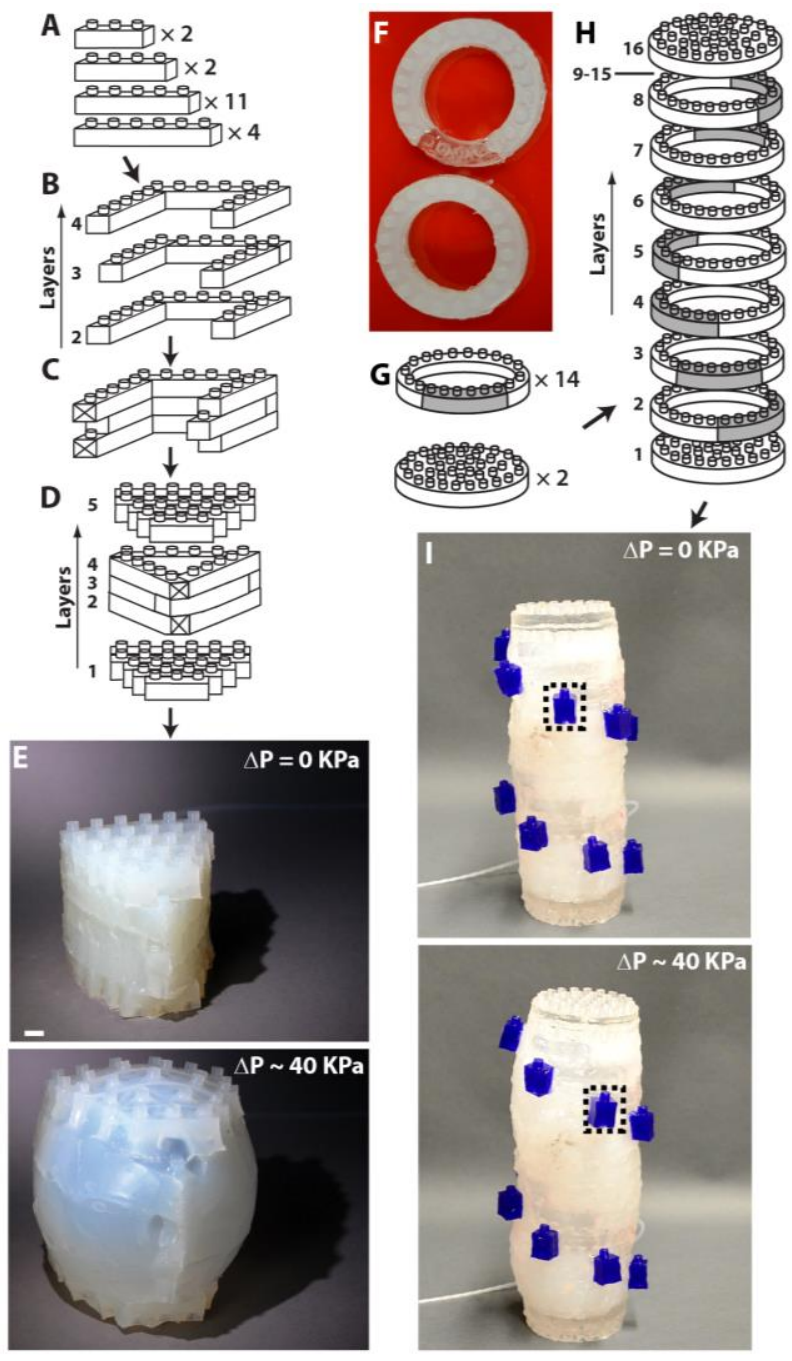


iterations of design useful to prototyping. Although we have focused on the mechanical properties of click-e-bricks important to the actuation of soft machines, fabrication using click-ebricks enables users to add or subtract other properties (e.g., optical, electrical, magnetic, etc.) to and from these structures by choosing (or fabricating) and combining click-e-bricks with desired attributes. The examples illustrated so far are centimeter-scale structures, but with further development to the size, geometry, and other properties of click-e-bricks-which certainly are not limited to the physical designs, or selections of materials, we have used in this demonstration - this strategy can be extended to fabrication at smaller or larger length scales.

This approach is a new direction in fabrication of soft structures that will be useful for soft machines, and more generally in those fields (e.g., digital ${ }^{[20,21,27]}$ or additive manufacturing ${ }^{[15,16]}$ ) concerned with the fabrication of 3D, composite structures. Click-e-brick assembly can be automated using existing technologies in these areas - assemblers to pick and place the click-ebricks and extruders to deposit glue - in order to overcome the limitations associated with each of the individual techniques, and to enable the rapid fabrication of structures, from click-e-bricks, of functional elastomeric structures. In the future it may also be possible to combine the concept of click-e-brick-based assembly with evolutionary algorithms that exploit non-linearities in the mechanics of these structures on inflation to arrive at optimized structures that are functional, though not necessarily intuitive. ${ }^{[23]}$

\section{Experimental Section}

Please see supporting information.

\section{Supporting Information}

Supporting Information is available from the Wiley Online Library or from the author.

\section{Acknowledgments}


The design and fabrication of elastomeric bricks and the soft structures based on them was supported by the US Department of Energy, Division of Materials Science, under award No. DEFG02-00ER45852, which also provided partial salary support for S.A.M, S.W.K, and J.L. The development of aspects related to soft machines was supported by the Defense Advanced Research Planning Agency (W911NF-11-1-0094), which also supported R.F.S. and A.A.S. Y.S. acknowledges the Natural Sciences and Engineering Research Council of Canada (NSERC) for support. Partial salary support for S.A.M, and S.W.K was provided by the Materials Research Science and Engineering Center at Harvard University (supported by the National Science Foundation under award number DMR-0820484.

Received: ((will be filled in by the editorial staff))

Revised: ((will be filled in by the editorial staff))

Published online: ((will be filled in by the editorial staff))

[1] F. Ilievski, A. D. Mazzeo, R. E. Shepherd, X. Chen, G. M. Whitesides, Angew. Chem. Int. Edit. 2011, 50, 1890.

[2] R. V. Martinez, C. R. Fish, X. Chen, G. M. Whitesides, Adv. Funct. Mater. 2012, 22, 1376.

[3] B. Mosadegh, P. Polygerinos, C. Keplinger, S. Wennstedt, R. F. Shepherd, U. Gupta, J. Shim, K. Bertoldi, C. J. Walsh, G. M. Whitesides, Adv. Funct. Mater. 2014, doi: 10.1002/adfm.201303288.

[4] J. C. McDonald, G. M. Whitesides, Accounts Chem. Res. 2002, 35, 491.

[5] K. Suzumori, Robot Auton. Syst. 1996, 18, 135.

[6] K. Suzumori, S. IIkura, H. Tanaka, 1991 IEEE Int. Conf. Rob. Autom. 1991, 204.

[7] R. F. Shepherd, F. Ilievski, W. Choi, S. A. Morin, A. A. Stokes, A. D. Mazzeo, X. Chen, M. Wang, G. M. Whitesides, Proc. Natl. Acad. Sci. U. S. A. 2011, 108, 20400.

[8] S. A. Morin, R. F. Shepherd, S. W. Kwok, A. A. Stokes, A. Nemiroski, G. M. Whitesides, Science 2012, 337, 828 . 
[9] R. V. Martinez, J. L. Branch, C. R. Fish, L. H. Jin, R. F. Shepherd, R. M. D. Nunes, Z. G. Suo, G. M. Whitesides, Adv. Mater. 2013, 25, 205.

[10] R. F. Shepherd, A. A. Stokes, J. Freake, J. Barber, P. W. Snyder, A. D. Mazzeo, L. Cademartiri, S. A. Morin, G. M. Whitesides, Angew. Chem. Int. Edit. 2013, 52, 2892.

[11] S. Kim, C. Laschi, B. Trimmer, Trends Biotechnol. 2013, 31, 287.

[12] S. W. Kwok, S. A. Morin, B. Mosadegh, J.-H. So, R. F. Shepherd, R. V. Martinez, B. Smith, F. C. Simeone, A. A. Stokes, G. M. Whitesides, Adv. Funct. Mater. 2013, doi: 10.1002/adfm.201303047.

[13] A. D. Marchese, C. D. Onal, D. Rus, Soft Robotics 2014, 1, 75.

[14] B. Wendel, D. Rietzel, F. Kuhnlein, R. Feulner, G. Hulder, E. Schmachtenberg, Macromol. Mater. Eng. 2008, 293, 799.

[15] H. Lipson, M. Kurman Fabricated: The New World of 3D Printing, John Wiley and Sons, Inc., Indianapolis, IN, USA 2013.

[16] J. A. Lewis, Adv. Funct. Mater. 2006, 16, 2193.

[17] E. Sachs, M. Cima, P. Williams, D. Brancazio, J. Cornie, J. Eng. Ind.-T. ASME 1992, 114, 481.

[18] Stratasys Ltd., Technical data provided by the manufacturer, http://www.stratasys.com/3dprinters/technology/polyjet-technology, accessed: March, 2014.

[19] Smooth-On, Technical data provided by the manufacturer, http://www.smoothon.com/tb/files/ECOFLEX_SERIES_TB.pdf, accessed: March, 2014.

[20] J. Hiller, H. Lipson, Rapid Prototyping J. 2009, 15, 137.

[21] J. Hiller, H. Lipson, Rapid Prototyping J. 2010, 16, 241.

[22] J. Hiller, H. Lipson, IEEE Trans. Robot. 2012, 28, 457.

[23] N. Cheney, R. MacCurdy, J. Clune, H. Lipson, in Proceedings of the 15th annual conference on Genetic and evolutionary computation, ACM, Amsterdam, Netherlands, 2013, 167.

[24] Y. N. Xia, G. M. Whitesides, Annu. Rev. Mater. Sci. 1998, 28, 153.

[25] C. Majidi, R. F. Shepherd, R. K. Kramer, G. M. Whitesides, R. J. Wood, Inter. J. Robot. Res. 2013, $32,1577$. 
[26] See supporting information for more detail.

[27] J. D. Hiller, J. Miller, H. Lipson, J. Microelectromech. Syst. 2011, 20, 1089. 